\title{
Kinesio Tape In Shoulder Rotator Cuff Tendinopathy: A Randomized, Blind Clinical Trial
}

\author{
L. Martins da Silva², G. Maciel Bello², B. Chuaste Flores², L. Silva Dias², P. Camargo², \\ L. F. Mengue ${ }^{2}$, V. Roque ${ }^{2}$, M. dos Santos Júnior ${ }^{2}$, I. Rodrigues ${ }^{1}$, G. Zanelatto ${ }^{1}$, M. B. \\ Dohnert $^{3}$, R. B. Daitx ${ }^{1}$ \\ 1 Physiotherapy Course, Lutheran University of Brazil, Torres, RS, Brazil \\ 2 Lutheran University of Brazil, Torres, RS, Brazil \\ 3 Teaching and Extension Laboratory in Orthopedic Trauma Physiotherapy (LAPEFITO), Gurupi University (UnirG), \\ Gurupi, Torres, Brazil
}

CORRESPONDING AUTHOR:

Marcelo Baptista Dohnert

1530 Gioás Street

apartment $900 \mathrm{~A}$

Bairro Centro 77410-010

Gurupi, Torres, Brazil

E-mail: mdohnert@hotmail.com

DOI:

10.32098/mltj.03.2020.03

LEVEL OF EVIDENCE: 1B

\begin{abstract}
SUMMARY
Background. Shoulder pain is a very common musculoskeletal disorder that affects many people, with rotator cuff (RC) injury as one of its main causes.

Objective. To analyze the efficacy of KT, both isolated and associated with exercise, on pain and function of patients with shoulder RC injuries.

Method. A randomized, blind clinical trial with sixty (60) participants with RC injuries, randomized into exercise group (EG), in which participants performed an exercise protocol; kinesio tape group (KTG), with application of the elastic bandage; and exercise + kinesio tape group (EKTG), in which participants performed both protocols. We evaluated pain intensity, active and passive mobility, muscle strength, and function.

Results. All intervention groups significantly improved pain, disability, and function. Regarding the latter, EKTG showed significantly greater improvements than EG and KTG $(\mathrm{p}<0.05)$. In addition, EKTG improved muscle strength in all evaluated movements. Groups EG and EKTG improved range of motion in all evaluated movements, for both the right and left shoulder.

Conclusions. Exercises were the basis of the treatment of RC injury. When associated with an exercise protocol, kinesio tape (KT) enhanced the effect of exercise in patients with shoulder RC injury. In isolation, KT was effective in reducing pain. Brazilian Clinical Trials Registry (REBEC) RBR-65qh7j.
\end{abstract}

\section{KEY WORDS}

Rotator cuff; kinesio tape; therapeutic exercise.

\section{BACKGROUND}

Shoulder pain is a very common musculoskeletal disorder that affects many people. ${ }^{1}$ It causes significant disability, and rotator cuff injury is one of its main risk factors $(2,3)$. The prevalence of shoulder pain ranges from 6.9 to $26.0 \%(1,4)$. Among the various conditions that may affect people with shoulder pain, subacromial impingement syndrome (SIS) has the highest prevalence and accounts for $36 \%$ of shoulder disorders (5). Subacromial impingement syndrome or SIS is a generic term used to define lesions affecting structures in the subacromial space, such as RC tendinosis, partial
$\mathrm{RC}$ rupture, and bursitis (1). Rotator cuff (RC) ruptures are a common cause of shoulder dysfunction and can lead to numerous functional deficits, including decreased range of motion (ROM), pain, and muscle weakness (6). Rotator cuff injuries and subacromial impingement are among the most common diagnoses in the shoulder region (7) being one of the most common causes of shoulder dysfunction (8). These events occur mainly in patients aged between 40 and 60 years and may be traumatic or degenerative (9). Their etiology is multifactorial $(10,11)$ and age is a factor that predisposes to tendon degeneration $(10,11)$. When associated with muscle 
overuse, repair does not occur properly, resulting in disorganization and tendon thickening (a process called tendinosis) (11).

Initial treatment of RC injuries is conservative and emphasizes rehabilitation programs as opposed to surgical interventions. ${ }^{2}$ Shoulder rehabilitation programs generally focus on strengthening shoulder and shoulder girdle (more specifically, RC) muscles (2). Several studies prove the effectiveness of an eccentric strengthening program for shoulder rotator cuff (12).

Kinesio tape (KT) is used with or without other physiotherapeutic interventions, especially to control pain, inflammation, and improve functional activity in patients with shoulder pain $(3,13)$. It is usually used as an adjunct in the rehabilitation of injuries $(3,13)$. After KT application, there is an increase in interstitial space leading to an increase in blood and lymphatic flow, with consequent decreased pressure on the underlying soft tissues. ${ }^{14}$ Kinesio tape may be an interesting option for improving shoulder neuromuscular control, with wide application in clinical practice (15). It has been argued that KT can reduce symptoms and functional limitations by improving proprioceptive feedback $(13,16)$. Moreover, KT has been reported to increase subacromial space in healthy individuals $(7,9)$.

Exercise programs that not only focus on scapular muscle strengthening but also emphasize motor control, including quality of movement, have been advocated for the treatment of individuals with RC injuries and included in shoulder sports injury prevention programs (17). Exercises play a fundamental role in the treatment of RC injuries (16). Studies show that several benefits can be obtained from physiotherapeutic exercises ${ }^{18,19}$ such as active and passive range-of-motion exercises, Codman pendulum exercises, stretching, and isometric and isotonic exercises. Eccentric exercises have been reported in the literature as the most recommended for treating degenerative tendon diseases (20). Eccentric training consists in the contraction of a muscle to control or slow down a load, while the muscle and tendon stretch or remain stretched (21). This technique has been recommended in the treatment of several pathologies such as Achilles tendon tendinopathy, patellar tendinopathy, lateral elbow epicondylitis, and RC (21). Other physiological processes that justify the use of eccentric exercises are increased fibroblast activity, accelerated collagen formation, formation and increase of type I collagen, and collagen organization and alignment (tendon remodeling) $(22,23)$.

This study is justified by the controversy found in the current literature on the effect of KT, either in isolation or associated with kinesiotherapeutic approaches, on general musculoskeletal disorders, especially in the treatment of partial injuries of shoulder rotator cuff.
Here, we evaluate the effects of KT, either in isolation or associated with exercise, on RC injuries. Variables assessed were: pain, joint mobility, muscle strength, and shoulder function in patients with RC injury.

\section{MATERIALS AND METHODS}

\section{Study design}

This was a randomized, blind clinical trial conducted at the Physiotherapy Clinic School of the Lutheran University of Brazil (Torres-RS) from August 2017 to June 2019. The study was registered in the Brazilian Clinical Trials Registry (REBEC) under the identification number RBR-65qh7j.

\section{Sample calculation}

The pain level assessed by VAS was used as the primary outcome to calculate sample size. Based on the study by Tantawy and Kamel (24), we estimated the mean and standard deviation of the initial pain of the study participants to be $4.9 \pm .1 .8$ in the experimental group and $4.8 \pm 1.6$ in the control group; and the mean and standard deviation of the final pain, i.e., after six weeks of treatment, to be $3.3 \pm 1.4$ for the eccentric training group and $4.1 \pm 1.5$ for the traditional training group. Using a study power of $80 \%$, a significance level of $95 \%$, and a sample size ratio of 1: 1: 1 (KT group: exercise group: KT + exercise group), we estimated 16 participants for each group, totaling 48 participants. Believing that losses and refusals would be around $20 \%$, we reached the final number of 20 participants for each group, totaling 60 participants.

\section{Sample randomization}

After the initial assessment, eligible participants were randomized through a list of random numbers provided by the EPI-Info ${ }^{\circledR}$ software. These participants chose a sealed envelope containing a random number corresponding to one of the intervention groups, previously defined by the list of random numbers. Participants were randomly divided into exercise group (EG), performing only one exercise protocol consisting of seven exercises; Kinesio tape group (KTG), where only the elastic bandage was applied to participants; and exercise + Kinesio tape group (EKTG), which performed both KT and exercise protocols, totaling 60 patients.

\section{Eligibility Criteria}

Eligibility criteria are shown in table I. 
Table I. Study inclusion and exclusion criteria.

\begin{tabular}{ll}
\hline Inclusion criteria & Exclusion criteria \\
\hline Patients aged 18 to 70 years & $\begin{array}{l}\text { Patients with history of shoulder dislocation and positive } \\
\text { displacement apprehension test }\end{array}$ \\
\hline Having signed the Informed Consent Form (ICF) & Intratendinous rotator cuff calcifications \\
\hline $\begin{array}{l}\text { Subacromial pain (grade } 3 \text { or more on VAS) for at least three } \\
\text { months }\end{array}$ & Proximal humeral fracture \\
$\begin{array}{l}\text { Two of three positive impingement tests (Jobe, Hawkins- } \\
\text { Kennedy, Neer) performed by an independent expert }\end{array}$ & Diagnosis of complete tendon rupture in the rotator cuff \\
\hline & Diagnosis of complete tendon rupture in the rotator cuff \\
\hline & Previous shoulder surgery \\
\hline Adhesive capsulitis \\
\hline & Type III acronym (Bigliani classification) on x-ray \\
\hline Use of corticoid infiltrates within three months prior to the study \\
\hline Rheumatic and neurological diseases \\
\hline $\begin{array}{l}\text { Patients with cognitive impairment that prevents them from } \\
\text { understanding the exercises }\end{array}$ \\
\hline $\begin{array}{l}\text { Patients with skin hypersensitivity to Kinesio tape or bandage } \\
\text { Patients undergoing or having undergone physiotherapy treatment } \\
\text { in the last 3 months }\end{array}$ \\
\hline
\end{tabular}

\section{DATA COLLECTION}

The evaluation protocol was performed at three moments. The initial evaluation was performed prior to randomization; the second evaluation after the end of the intervention; and the third (follow-up) at 90 days after the end of the intervention protocol. Evaluations were performed by an independent, previously trained, blind evaluator (who did not know which group the research participant belonged to).

\section{Anthropometric evaluation}

Body mass index (BMI) was calculated by measuring body height and weight.

\section{Pain intensity assessment}

Pain intensity was measured using the Visual Analogue Scale (VAS). In this scale, the patient is asked about his/her pain level on a scale from zero to ten, where zero means total absence of pain and ten the maximum tolerable pain (25). The intensity of pain at rest was assessed 24 hours prior to evaluation.

\section{Muscle strength assessment}

Maximal voluntary isometric contraction (MVIC) was evaluated by manual dynamometry. Muscle strength of the rotators and deltoid was measured using a Chattanooga ${ }^{\circledR}$ push-pull dynamometer. The peak of maximum voluntary isometric force was measured at the average arc angle of each movement. The average of two of the three measurements taken was used as the maximum voluntary isometric contraction (MVIC), as described by Ling et al. (26).

For flexion, abduction, and extension movements, the participant remained standing. The shoulder was oriented at $0^{\circ}$. The dynamometer was positioned above the elbow, and the resistance applied perpendicular to the distal humerus, one centimeter above the lateral epicondyle. The participant was asked to apply a force contrary to the position of the dynamometer. Participants maintained the positions mentioned while the examiner applied the force through the dynamometer with the participant accumulating maximum tension in one to two seconds and maintaining tension for four to five seconds.

External and internal rotation movements were tested with the participant lying supine, shoulder at $90^{\circ}$ abduction and $90^{\circ}$ elbow flexion. The dynamometer was positioned one centimeter below the wrist, perpendicular to the dorsal distal forearm, and one centimeter proximal to the ulnar styloid. The participant was asked to apply a force contrary to the position of the dynamometer. Two measurements were taken for each strength test, with a 30 -second rest between measurements to allow muscle recovery. The average of the two measurements of each strength test was used for data analysis. 


\section{Range of motion assessment}

Active and passive movements of flexion, abduction, extension, internal and external rotation were measured using a universal goniometer for determining ROM. To assess shoulder joint flexion and extension mobility, the participant remained standing. The goniometer axis was positioned centrally at the glenohumeral joint, with the fixed arm perpendicular to the ground, and the movable arm positioned in line with the axis of the participant's arm. For abduction, the central axis of the goniometer was positioned in the posterior glenohumeral joint. To assess rotation, the participant was placed in the supine position with the shoulder abducted at $90^{\circ}$ and the elbow flexed at $90^{\circ}$. The central axis of the goniometer was positioned over the olecranon of the ulna. The fixed arm was positioned perpendicular to the ground, while the movable arm was positioned in line with the patient's forearm.

\section{FUNCTION ASSESSMENT}

Data regarding function and disability were collected through function and quality of life questionnaires (UCLA (27), modified) and the Shoulder Pain and Disability Index (SPADI) (28). The University of California, Los Angeles (UCLA) scale assesses pain intensity, shoulder function, active range of motion, muscle strength, and patient satisfaction. The higher the score obtained, the better the patient's functional level. A score of $34-35$ points corresponds to excellent results, $28-33$ points correspond to good results, 21-27 points correspond to reasonable results, and $0-20$ points correspond to bad results. The Constant-Murley score assesses pain and disability in patients with shoulder disorders. It has two subjective parameters (pain and daily living activities) and two objective parameters (range of motion and muscle strength). The scale totals 100 points, of which 35 are allocated to subjective parameters and 65 to objective parameters. Finally, the Shoulder Pain and Disability Index (SPADI) is a quality of life questionnaire designed to assess pain and disability in shoulder disor- ders. It consists of thirteen items with two dimensions, one for pain and the other for functional activities. The final questionnaire score obtained separately for each dimension is converted into percentages ranging from zero to one hundred, with the highest score indicating the worst condition of shoulder dysfunction.

\section{EXERCISE PROTOCOL}

For participants in the exercise group, the intervention protocol consisted of a program with seven eccentric exercises. The program lasted four weeks and the sessions were held three times a week. As the patient became more familiar with the program and progressed in mobility and strength weekly, the load of each exercise was increased by $0.5 \mathrm{~kg}$ per week until reaching $1 \mathrm{~kg}$; from the last week until the end of the intervention protocol, the load was $2 \mathrm{~kg}$. The increase in weekly load was suspended only in case of maintenance of pain on movement or lack of strength to progress further (table II).

\section{KT intervention}

Kinesio tape was applied according to the protocol for RC tendinitis/shoulder impingement suggested by Kase (18). For groups KTG and EKTG, KT was applied twice a week for four weeks and was replaced every three days. Each participant used three tapes. The first tape (Y-shaped) was applied over the supraspinatus and infraspinatus muscles, anchored towards their origin (18). The tape was applied using a 15 to $25 \%$ tension, with the participant in a contralateral inclination of the cervical spine to the shoulder, and the arm in internal rotation at the back (figure 1). The second tape (also Y-shaped) was applied bypassing the deltoid muscle belly, with a 15 to $25 \%$ tension, also towards the origin (18). A portion of the tape bypassed the anterior part of the deltoid, with the arm in horizontal abduction and in external rotation. The other portion of the tape bypassed the posterior aspect of the posterior deltoid, being applied with the arm

Table II. Exercise program.

Exercise 1. Sitting. Performs shoulder/scapular elevation up to $90^{\circ}$ (15 repetitions).

Exercise 2. Sitting. Performs internal rotation as if scratching the back (15 repetitions).

Exercise 3. In lateral decubitus, arm supported, elbow at $90^{\circ}$, performs external rotation (15 repetitions).

Exercise 4. In lateral decubitus, arm supported, performs internal rotation (15 repetitions).

Exercise 5. In supine position, performs shoulder/scapular elevation up to $90^{\circ}$ (15 repetitions).

Exercise 6. In prone position, with one arm off the examination table, performs extension movement (one-arm rowing exercise) (15 repetitions).

Exercise 7. In orthostasis, performs wall push-ups (15 repetitions). 
in horizontal adduction and in internal rotation, as if touching the contralateral hip (figure 1) (18). Finally, the third tape (I-shaped) anchored the other two from the coracoid process towards the scapular spine, using a tension of 50 to $75 \%$, with upper limb flexion and slight horizontal adduction (figure 1) (18). All participants were monitored for signs of hypersensitivity or allergy to KT. In the presence of these signs, the bandage would be removed and the patient referred for medical evaluation at the health network.

\section{STATISTICAL ANALYSIS}

Statistical Package for Social Sciences (SPSS) version 23.0 was used for data analysis. Data were initially expressed as frequency, mean, median and standard deviation. Afterwards, the normality of data distribution was analyzed using the Shapiro-Wilk test.

For intragroup comparisons (EG, KTG, and EKTG) at the various times of the study, parametric data were statistically analyzed by one-way analysis of variance (ANOVA) for repeated measures followed by Bonferroni post-hoc test. For intergroup comparisons, one-way analysis of variance (ANOVA) was used followed by Tukey post-hoc test, both at pre- and postintervention and 90 days after the intervention protocol. For nonparametric variables between groups, the Kruskal-Wallis and Friedman tests were used for intragroup analysis. The significance level established for all statistical tests was $\mathrm{p}<0.05$.

\section{RESULTS}

The initial sample consisted of 60 participants randomly assigned to exercise group (EG) $(n=20)$, Kinesio tape group $($ KTG) $(n=20)$, and exercise + Kinesio tape group (EKTG) $(n=20)$. All participants completed the final evaluation. However, in the follow-up assessment, there were two losses in EG, one for withdrawal and one participant who was referred for surgery. Moreover, EKTG had a loss due to arm fracture. No side effects were observed regarding the use of $\mathrm{KT}$ or the progression of the proposed exercises in the study participants. Thus, 57 patients completed the study (figure 1).

Table III shows the initial characteristics of the sample. The groups were homogeneous regarding the analyzed variables. All intervention groups improved pain after the intervention and maintained this reduction in the follow-up assessment. There were no significant differences between groups (figure 3).

Considering UCLA scores, all groups significantly improved function after the intervention. In the follow-up assessment, EKTG showed a significantly higher score than KTG $(\mathrm{p}=0.022)$ (figure 4).

All groups also significantly improved disability from baseline to endpoint, as assessed by the SPADI-Brazil questionnaire. In the follow-up assessment, EKTG showed a significantly higher score than KTG ( $\mathrm{p}=0.041)$ and EG $(\mathrm{p}<0.024)$ (figure 5).

The exercise + Kinesio tape group (EKTG) significantly increased right shoulder mobility $(\mathrm{p}<0.05)$. For this group, flexion and internal rotation range of motion were significantly higher compared to KTG $(\mathrm{p}=0.02)$. The exercise group (EG) significantly increased active ROM of flexion, abduction, and external and internal rotation at the end of the protocol $(\mathrm{p}<0.05)$. The Kinesio tape group $(\mathrm{KTG})$ improved only abduction and external rotation ROM $(\mathrm{p}<0.05)$ (table IV).

Participants with left shoulder pain in the EKTG improved all active ROM $(\mathrm{p}<0.05)$. For EG participants,

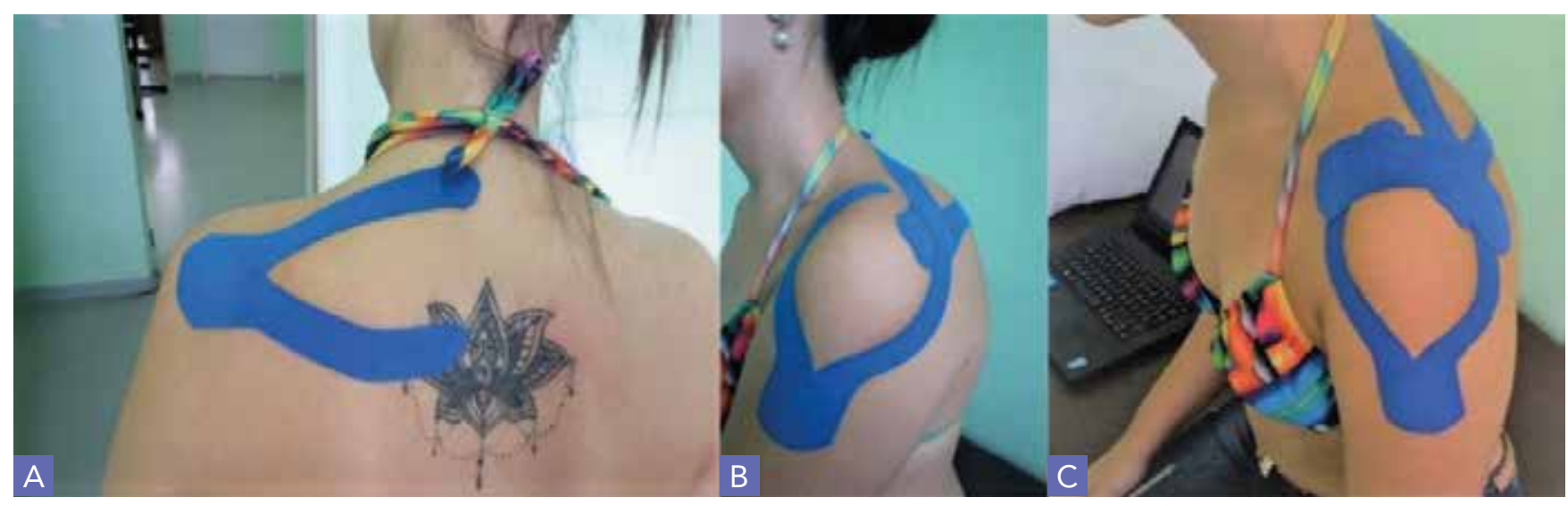

Figure 1. Application of Y-shaped Kinesio tape in the scapula (A); Y-shaped Kinesio tape in the deltoid (B); and I-shaped Kinesio tape for anchorage and scapulohumeral joint positioning (C). 


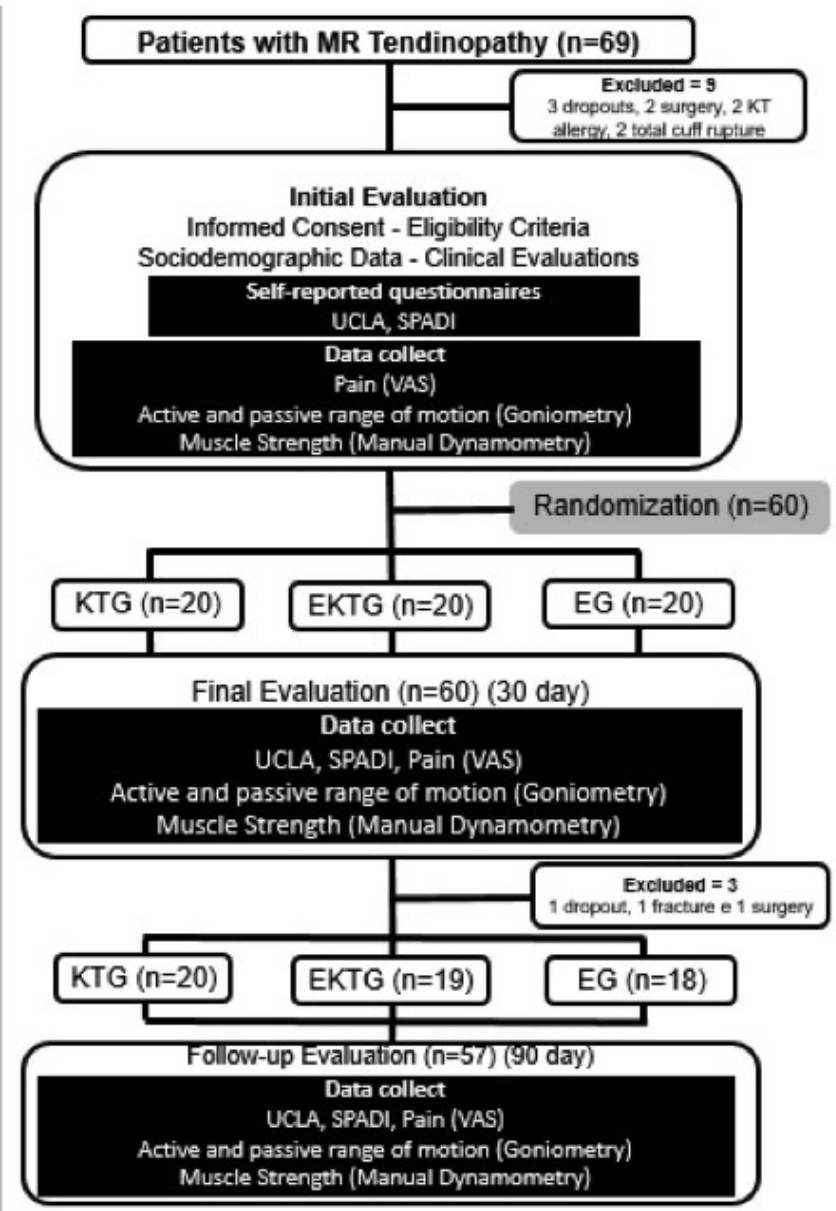

Figure 2. Study flowchart.

only extension ROM did not significantly increase after the intervention. In turn, KTG participants increased only flexion and abduction ROM $(\mathrm{p}<0.05)$ (table V). There were no differences between groups in the different evaluation times.

The muscle strength of patients with right shoulder pain, represented by the maximum voluntary isometric contraction (MVIC), did not differ significantly between groups in the different moments of the study. The Kinesio tape group (KTG) significantly improved only flexion and external rotation ROM of the right shoulder $(\mathrm{p}<0.05)$ at the end of the protocol. The exercise + Kinesio tape group (EKTG) increased muscle strength in flexion $(\mathrm{p}<0.05)$, external rotation $(\mathrm{p}<0.01)$, and internal rotation $(\mathrm{p}<0.01)$ $\mathrm{ROM}$ of the right shoulder at the end of the protocol. However, muscle strength increased significantly for all ROM from baseline to follow-up $(\mathrm{p}<0.05)$. Participants in the EG significantly increased strength in flexion, abduc- tion, and internal rotation $\mathrm{ROM}$ of the right shoulder at the end of the intervention $(\mathrm{p}<0.05)$. For flexion ROM, this strength gain remained at the follow-up assessment $(\mathrm{p}<0.05)($ table VI).

The muscle strength of patients with left shoulder pain did not differ significantly between groups at the different times of the study. The Kinesio tape group (KTG) significantly increased muscle strength for external rotation only at the follow-up assessment $(\mathrm{p}<0.05)$. Participants in the EKTG significantly improved strength from baseline to endpoint only in external rotation ROM of the left shoul$\operatorname{der}(\mathrm{p}<0.05)$. The exercise group $(\mathrm{EG})$, in turn, did not significantly improve left shoulder ROM (table VII).

Table III. Sociodemographic characteristics of the sample $(n=60)$.

\begin{tabular}{|c|c|c|c|c|}
\hline \multicolumn{5}{|c|}{ Intervention Group } \\
\hline & $\begin{array}{c}\text { KTG } \\
(n=20)\end{array}$ & $\begin{array}{l}\text { EKTG } \\
(n=20)\end{array}$ & $\begin{array}{c}\text { EG } \\
(n=20)\end{array}$ & p-value \\
\hline Age, years $( \pm \mathrm{sd})$ & $\begin{array}{c}48.65 \pm \\
10.27\end{array}$ & $\begin{array}{c}46.95 \pm \\
10.74\end{array}$ & $\begin{array}{c}49.20 \pm \\
13.13\end{array}$ & $0.88^{\#}$ \\
\hline Gender, n (\%) & & & & $0.37 \$$ \\
\hline Male & $6(30.0)$ & $8(40.0)$ & $4(20.0)$ & \\
\hline Female & $14(70.0)$ & $12(60.0)$ & $16(80.0)$ & \\
\hline Skin color, n (\%) & & & & $0.15^{\$}$ \\
\hline White & $17(85.0)$ & $19(95.0)$ & $8(100.0)$ & \\
\hline Black & $3(15.0)$ & $1(5.0)$ & $0(0.0)$ & \\
\hline $\begin{array}{l}\text { Affected } \\
\text { shoulder, n (\%) }\end{array}$ & & & & $0.58^{\$}$ \\
\hline Right & $9(45.0)$ & $10(50.0)$ & $6(30.0)$ & \\
\hline Left & $7(35.0)$ & $7(35.0)$ & $7(35.0)$ & \\
\hline Both & $4(20.0)$ & $3(15.0)$ & $7(35.0)$ & \\
\hline Occupation & & & & $0.14^{\$}$ \\
\hline $\begin{array}{l}\text { General } \\
\text { services }\end{array}$ & $4(20.0)$ & $0(0.0)$ & $1(5.0)$ & \\
\hline Housekeeper & $2(10.0)$ & $6(30.0)$ & $3(15.0)$ & \\
\hline Bricklayer & $2(10.0)$ & $0(0.0)$ & $0(0.0)$ & \\
\hline Cook & $2(10.0)$ & $0(0.0)$ & $0(0.0)$ & \\
\hline Others & $10(50.0)$ & $14(70.0)$ & $16(80.0)$ & \\
\hline $\begin{array}{l}\text { Time of pain, } \\
\text { years }( \pm \mathrm{sd})\end{array}$ & $\begin{array}{c}4.95 \pm \\
5.63\end{array}$ & $\begin{array}{c}5.00 \pm \\
8.05\end{array}$ & $\begin{array}{c}4.88 \pm \\
8.99\end{array}$ & $0.98^{\#}$ \\
\hline
\end{tabular}

Legend: kg: kilogram; cm: centimeters; KTG: Kinesio tape group; EKTG: exercise + Kinesio tape group; EG: exercise group.

\# One-way ANOVA.

$\$$ Chi-square. 


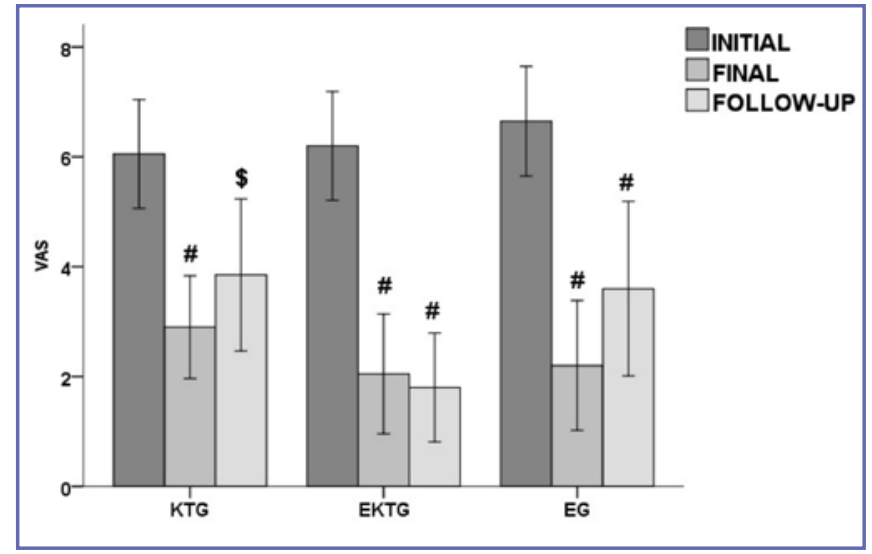

Figure 3. Pain level (VAS) in study groups $(n=60)$ $\# p=0.001$ compared to baseline. ANOVA for repeated measures. $\$ p=0.02$ compared to baseline. ANOVA for repeated measures.

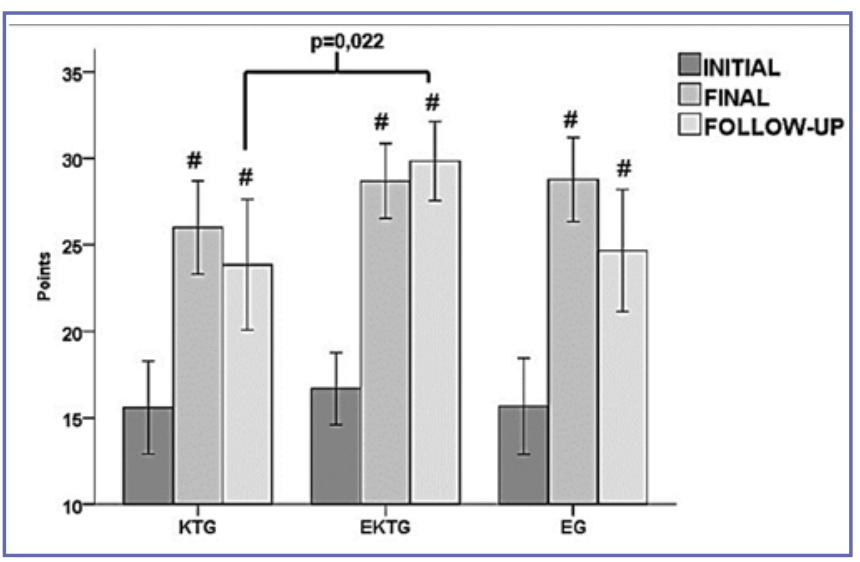

Figure 4. UCLA questionnaire score in study groups $(n=60)$ $\# p=0.001$ compared to baseline. ANOVA for repeated measures.

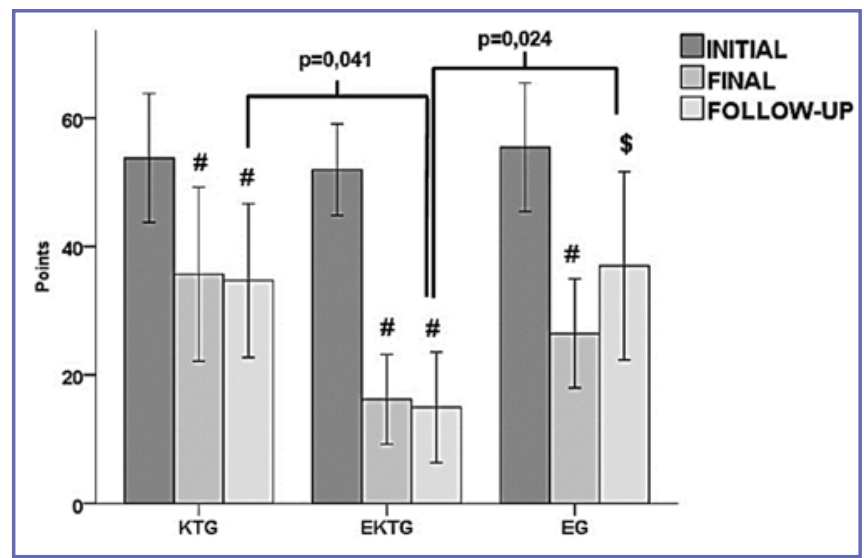

Figure 5. Total SPADI questionnaire score in study groups $(n=60)$. $\# p=0.001$ compared to baseline. Student $t$ test. $\$ p<0.05$ compared to KTG. One-way ANOVA.

\section{DISCUSSION}

The present study evaluated the efficacy of KT, both isolated and associated with exercise, on pain, disability, mobility, and strength in subjects with RC injury. Oliva et al. (29), in their guideline about rotator cuff tears, report that the exercise demonstrates some advantages, in an isolated or associated way in an Individual Rehabilitation Project (PRI) in patients with rotator cuff tears. Pain intensity decreased in all study groups from baseline to endpoint and follow-up. This result is corroborated by the study by Kocyigit et al., (30) who investigated the effect of KT on pain intensity, strength, and mobility in 41 patients with RC injury allocated to KT group $(\mathrm{n}=21)$ and placebo KT group $(\mathrm{n}=20)$. The authors prescribed a Codman pendulum exercise program with three sets of 10 repetitions per day for all patients (30). The experimental KT group significantly reduced pain intensity (30). The authors concluded that KT immediately improved pain in all patients with RC injury (30). Kinesio tape, in conjunction with other physiotherapy interventions, can facilitate or inhibit muscle function, maintain joint positioning, reduce pain, and provide proprioceptive feedback to achieve and maintain body alignment (31). Increased proprioceptive feedback by sensory stimulation from KT application may strengthen postural control and facilitate the return to previous muscle activity (32). Another probable explanation of the effect of KT on pain modulation relates to the pain gate theory, where it is suggested that the bandage stimulates neuromuscular pathways. This mechanical stimulation provided by KT activates fast conducting fibers performing synapses with inhibitory interneurons, which closes the pain gate, not allowing the passage of nociceptive stimuli $(33,34)$. Micinilli et al. approached 21 patients with rotator cuff tendinopathy using real KT and sham KT. Both groups performed the same exercises protocol. The authors observed that KT can facilitate the immediate reduction of pain during rehabilitation, probably due to the mechanism of the pain gate, which KT produces sensory stimulus, which reduces the nociceptive signal (35). Similarly, Aguilar-Ferrandiz et al. (36) investigated the effect of KT and placebo KT application in women with chronic venous insufficiency. One hundred and twenty postmenopausal women with mild to moderate chronic venous insufficiency were randomly assigned to GK $(\mathrm{n}=60)$ group, who received standardized KT treatment (15 to $50 \%$ tension) for gastrocnemius muscle enhancement and ankle functional correction; and placebo KT group $(\mathrm{n}=60)$ (36). Pain level and overall health were recorded at baseline and after four weeks of treatment (36). The authors concluded that KT therapy improves pain symptoms and peripheral venous flow and may slightly increase overall health in women with chronic venous insufficiency for up to four weeks after treatment (36). 
Table IV. Active range of motion (ROM) of participants with right shoulder pain $(n=39)$.

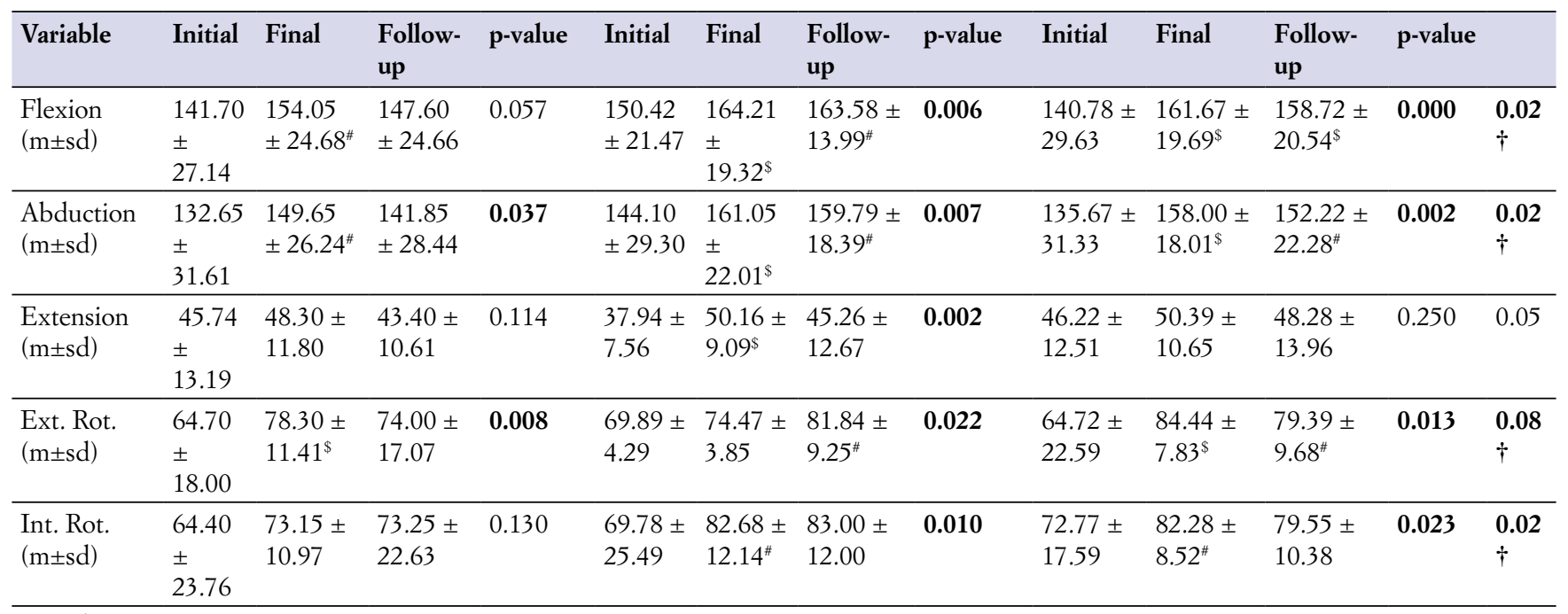

Legend:

$\$ \mathrm{p}<0.005$ compared to the baseline of the same group. One-way ANOVA for repeated measures.

\# $\mathrm{p}<0.05$ compared to the baseline of the same group. One-way ANOVA for repeated measures.

$\dagger \mathrm{p}=0.02$ between EKTG and KTG. One-way ANOVA.

Bold data indicate significant values.

Table V. Active range of motion (ROM) of participants with left shoulder pain. $(n=35)$.

\begin{tabular}{|c|c|c|c|c|c|c|c|c|c|c|c|c|c|}
\hline & $\begin{array}{l}\text { KTG } \\
(n=11)\end{array}$ & & & & & $\begin{array}{l}\text { EKTG } \\
(n=10)\end{array}$ & & & $\begin{array}{l}\text { EG } \\
(n=14)\end{array}$ & & & & $\mathrm{P}$-value \\
\hline Variable & Initial & Final & $\begin{array}{l}\text { Follow- } \\
\text { up }\end{array}$ & p-value & Initial & Final & $\begin{array}{l}\text { Follow- } \\
\text { up }\end{array}$ & p-value & Initial & Final & $\begin{array}{l}\text { Follow- } \\
\text { up }\end{array}$ & p-value & \\
\hline $\begin{array}{l}\text { Flexion } \\
(\mathrm{m} \pm \mathrm{sd})\end{array}$ & $\begin{array}{l}143.45 \\
\pm 21.43\end{array}$ & $\begin{array}{l}159.05 \\
\pm 18.14^{\#}\end{array}$ & $\begin{array}{l}161.00 \pm \\
15.39^{\$}\end{array}$ & 0.009 & $\begin{array}{l}1148.95 \\
\pm 21.77\end{array}$ & $\begin{array}{l}163.94 \\
\pm 15.28^{\$}\end{array}$ & $\begin{array}{l}167.74 \pm \\
13.58^{\$}\end{array}$ & 0.001 & $\begin{array}{l}148.28 \\
\pm 23.86\end{array}$ & $\begin{array}{l}165.78 \\
\pm 14.21^{\$}\end{array}$ & $\begin{array}{l}159.28 \pm \\
24.08\end{array}$ & 0.001 & 0.95 \\
\hline $\begin{array}{l}\text { Extension } \\
(\mathrm{m} \pm \mathrm{sd})\end{array}$ & $\begin{array}{l}48.15 \pm \\
11.86\end{array}$ & $\begin{array}{l}50.80 \pm \\
7.90\end{array}$ & $\begin{array}{l}49.20 \pm \\
10.70\end{array}$ & 0.234 & $\begin{array}{l}39.79 \pm \\
8.61\end{array}$ & $\begin{array}{l}50.63 \pm \\
9.21^{\$}\end{array}$ & $\begin{array}{l}50.00 \pm \\
11.40^{\$}\end{array}$ & 0.000 & $\begin{array}{l}45.94 \pm \\
10.36\end{array}$ & $\begin{array}{l}48.78 \pm \\
11.62\end{array}$ & $\begin{array}{l}50.94 \pm \\
11.05\end{array}$ & 0.175 & 0.67 \\
\hline $\begin{array}{l}\text { Ext. Rot. } \\
(\mathrm{m} \pm \mathrm{sd})\end{array}$ & $\begin{array}{l}67.80 \pm \\
16.54\end{array}$ & $\begin{array}{l}76.45 \pm \\
11.38\end{array}$ & $\begin{array}{l}74.65 \pm \\
14.56\end{array}$ & 0.068 & $\begin{array}{l}69.42 \pm \\
15.40\end{array}$ & $\begin{array}{l}76.53 \pm \\
15.84\end{array}$ & $\begin{array}{l}81.84 \pm \\
9.25\end{array}$ & 0.002 & $\begin{array}{l}77.33 \pm \\
13.78\end{array}$ & $\begin{array}{l}88.67 \pm \\
9.89^{\$}\end{array}$ & $\begin{array}{l}86.06 \pm \\
5.99^{\#}\end{array}$ & 0.005 & 0.31 \\
\hline $\begin{array}{l}\text { Int. Rot. } \\
(\mathrm{m} \pm \mathrm{sd})\end{array}$ & $\begin{array}{l}72.96 \pm \\
20.39\end{array}$ & $\begin{array}{l}80.90 \pm \\
13.76\end{array}$ & $\begin{array}{l}79.85 \pm \\
19.79\end{array}$ & 0.183 & $\begin{array}{l}75.84 \pm \\
27.69\end{array}$ & $\begin{array}{l}91.32 \pm \\
18.47^{\$}\end{array}$ & $\begin{array}{l}88.63 \pm \\
8.53\end{array}$ & 0.000 & $\begin{array}{l}63.61 \pm \\
23.45\end{array}$ & $\begin{array}{l}81.89 \pm \\
13.96^{\$}\end{array}$ & $\begin{array}{l}78.11 \pm \\
11.30^{\#}\end{array}$ & 0.007 & 0.82 \\
\hline
\end{tabular}

$\$ p<0.005$ compared to the endpoint of the same group. One-way ANOVA for repeated measures.

\# $\mathrm{p}<0.05$ compared to the baseline of the same group. One-way ANOVA for repeated measures.

Bold data indicate significant values

The UCLA scale showed an increase in functional and quality of life scores from baseline to endpoint in all groups of the present study. For these variables, EKTG demonstrated a better outcome than KTG in the follow-up assessment. Corroborating these results, Şahin et al. (37) evaluated 99 patients with RC injury, assigned to nonsteroidal anti-inflam- matory group ( $\mathrm{n}=33)$; KT + nonsteroidal anti-inflammatory group ( $\mathrm{n}=33$ ); and subacromial corticosteroid injection + nonsteroidal anti-inflammatory group $(n=33)$. All three groups showed significant improvements after intervention (37). However, the KT + nonsteroidal anti-inflammatory group and the subacromial corticosteroid injection + nonste- 
Table VI. Muscle strength (MVIC) of participants with right shoulder pain $(n=39)$.

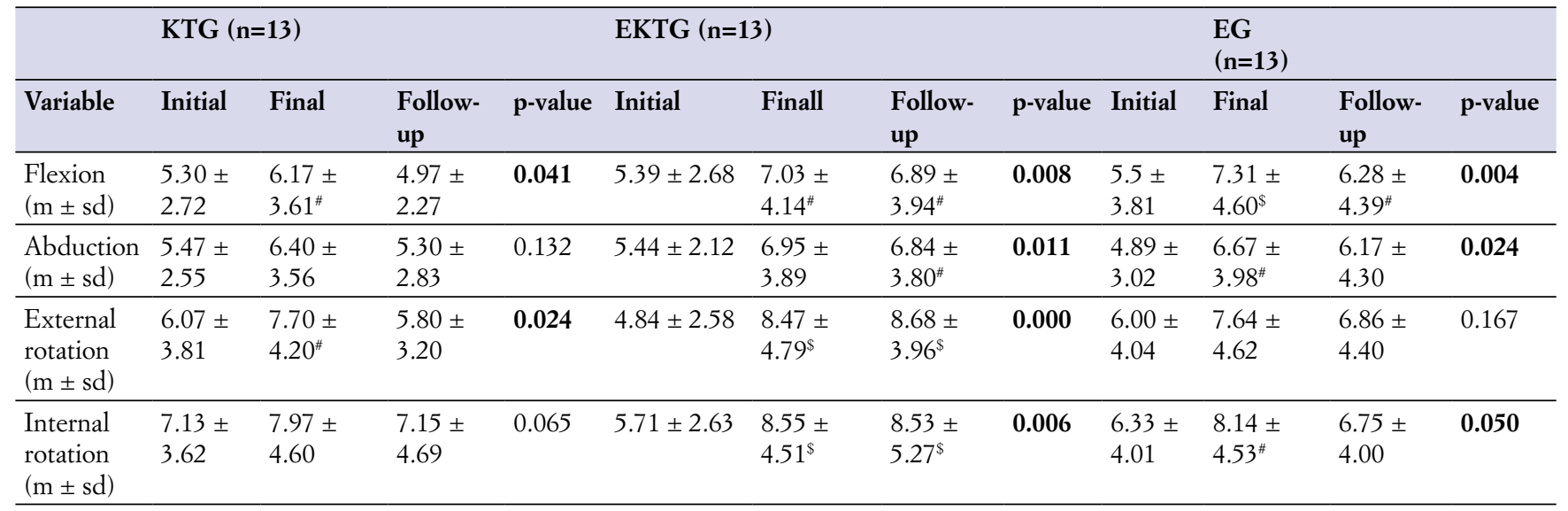

\# $\mathrm{p}<0.05$ compared to the baseline of the same group. One-way ANOVA for repeated measures.

$\$ p<0.01$ compared to the endpoint of the same group. One-way ANOVA for repeated measures.

Bold data indicate significant values.

Table VII. Muscle strength (MVIC) of participants with left shoulder pain $(n=35)$.

\begin{tabular}{|c|c|c|c|c|c|c|c|c|c|c|c|c|}
\hline \multirow[b]{2}{*}{ Variable } & \multicolumn{4}{|c|}{ KTG $(n=11)$} & \multicolumn{8}{|c|}{ EKTG $(n=10)$} \\
\hline & Initial & Final & $\begin{array}{l}\text { Follow- } \\
\text { up }\end{array}$ & p-value & Initial & Final & $\begin{array}{l}\text { Follow- } \\
\text { up }\end{array}$ & p-value & Initial & Final & Follow-up & p-value \\
\hline $\begin{array}{l}\text { Abduction, } \\
(\mathrm{m} \pm \mathrm{sd})\end{array}$ & $\begin{array}{l}5.92 \pm \\
3.01\end{array}$ & $\begin{array}{l}7.00 \pm \\
3.40 \\
\end{array}$ & $\begin{array}{l}6.35 \pm \\
3.29 \\
\end{array}$ & 0.069 & $\begin{array}{l}5.68 \pm \\
2.13 \\
\end{array}$ & $5.69 \pm 2.92$ & $\begin{array}{l}7.16 \pm \\
3.33 \\
\end{array}$ & 0.162 & $\begin{array}{l}4.77 \pm \\
3.05 \\
\end{array}$ & $\begin{array}{l}6.03 \pm \\
3.99 \\
\end{array}$ & $\begin{array}{l}5.81 \pm \\
4.01 \\
\end{array}$ & 0.230 \\
\hline $\begin{array}{l}\text { Internal } \\
\text { rotation (m } \\
\pm \mathrm{sd})\end{array}$ & $\begin{array}{l}7.58 \pm \\
3.69\end{array}$ & $\begin{array}{l}8.20 \pm \\
4.54\end{array}$ & $\begin{array}{l}7.88 \pm \\
4.98\end{array}$ & 0.367 & $\begin{array}{l}6.84 \pm \\
4.35\end{array}$ & $8.34 \pm 4.98$ & $\begin{array}{l}9.32 \pm \\
4.94\end{array}$ & 0.080 & $\begin{array}{l}6.50 \pm \\
4.14\end{array}$ & $\begin{array}{l}7.28 \pm \\
3.38\end{array}$ & $\begin{array}{l}7.75 \pm \\
4.28\end{array}$ & 0.175 \\
\hline
\end{tabular}

\# $\mathrm{p}<0.05$ compared to the baseline of the same group. One-way ANOVA for repeated measures.

$\$ p<0.005$ compared to the baseline of the same group. One-way ANOVA for repeated measures.

$\& \mathrm{p}<0.05$ compared to the endpoint. One-way ANOVA for repeated measures.

Bold data indicate significant values.

roidal anti-inflammatory group showed a greater tendency for improvement in all variables. The authors then concluded that the addition of KT or subacromial corticosteroid injection to treatment with anti-inflammatory drugs seems to have a significant and similar effect in patients with RC injuries (37). Therefore, KT may serve as an alternative treatment when corticosteroid injection is contraindicated (37). The SPADI-Brazil questionnaire showed statistically significant results for functional and quality of life scores in all study groups. However, EKTG showed a statistically significant improvement at follow-up when compared to EG and
KTG. Kaya et al. addressed 54 patients diagnosed with SIS allocated to $\mathrm{KT}+$ exercise group $(\mathrm{n}=28)$ and manual therapy + exercise group $(n=26)$ (38). Shoulder pain and functionality were evaluated (38). After intervention, there were significant differences in both groups in terms of decreased pain and improved disability, demonstrating that exercise is the basis for improvement in these patients (36). However, only the KT + exercise group showed a significant reduction in nighttime pain (38). Simsek et al. (2013) conducted a similar study evaluating strength, pain, ROM, and disability on the $5^{\text {th }}$ and $12^{\text {th }}$ day of treatment of 38 subjects with SIS, 
assigned to KT + exercise group $(\mathrm{n}=19)$ and placebo $\mathrm{KT}+$ exercise group $(\mathrm{n}=19)$ (39). Both groups improved scores at the $5^{\text {th }}$ and $12^{\text {th }}$ day of evaluations $(\mathrm{p}<0.05)$. In comparisons between groups, movement pain and disability score improved significantly in the KT + exercise group on day 5 ( $\mathrm{p}<0.01$ ) (39). Nighttime pain, movement pain, disability score, shoulder external rotation strength, and pain-free shoulder abduction ROM improved significantly in the KT + exercise group on the $12^{\text {th }}$ day $(\mathrm{p}<0.05)$ (39). However, passive shoulder flexion ROM increased more in the placebo KT + exercise group on the $12^{\text {th }}$ day $(\mathrm{p}<0.05)$ (39). The authors suggest that the reduction of pain and disability in the KT + exercise group occurred due to traction generated by the tension applied along the tape in the area of interest, thus increasing blood flow and producing neurophysiological effects to prevent pain transmission through the mechanism proposed in the pain gate theory (39). Notwithstanding, KT has shown effective short-term results (39). The authors concluded that the addition of KT to the exercise program appeared to be more effective than the exercise program alone for treating SIS (39).

Range of motion (ROM) in the affected shoulder showed that EKTG improved all evaluated movements. Corroborating these findings, Subaşı et al. (40) investigated the efficacy of KT compared to subacromial injection of betamethasone and prilocaine associated with a three-month exercise program, including stretching and strengthening exercises in 70 patients with RC injury (40). Participants were randomly assigned to injection group $(\mathrm{n}=35)$ and $\mathrm{KT}$ group $(\mathrm{n}=35)(40)$. Kinesio tape (KT) was applied three times for five consecutive days, with a two-day interval between applications (40). All patients were evaluated at baseline and one and three months after intervention (40). Significant differences were detected in pain and SPADI scores, as well as in ROM measurements in both groups (40). No significant differences were detected between groups, except for the degree of active flexion in favor of the KT group(40). Together with an exercise program, both KT and steroid injection therapy have been found to be effective in treating RC injuries. Thus, KT may be an alternative treatment option in the rehabilitation of RC injuries, especially when a noninvasive technique is required (40).

Muscle strength of the affected right shoulder showed statistically significant differences in KTG only in flexion and external rotation measurements. In turn, EKTG participants showed a statistically significant improvement in all movements. Finally, EG improved all movements at endpoint, except for external rotation. Groups KTG and EKTG improved muscle strength of the affected left shoulder in external rotation only. In contrast, EG did not improve any of the movements. The improvement in muscle strength, most significant in the KT group, can be justified by the action of $\mathrm{KT}$ in facilitating muscle activity and in the immediate increase of muscle strength, which may be due to concentric traction of the fascia (35). In a crossover study, Hsu et al.(41). compared the effects of therapeutic KT (Y-shaped tape with $25 \%$ tension) and placebo KT (Y-shaped tape without tension) in baseball players with RC injuries in two weekly sessions with three-day intervals (41). Three-dimensional scapular movements and electromyographic (EMG) activities of the upper and lower trapezius and anterior serratus muscle were measured during shoulder elevation with a $2-\mathrm{kg}$ load and were performed three consecutive times to collect electromyographic data (41). Lower trapezius strength was tested before and after each KT application (41). Results showed that the therapeutic KT group significantly increased $30^{\circ}$ and $60^{\circ}$ posterior scapular inclination during arm elevation (41). Moreover, this group increased lower trapezius muscle activity in the $60-30^{\circ}$ arm adduction compared to placebo KT (41). These improvements led to positive changes in scapular movement and muscle performance (41). The results supported the use of KT as an adjunct to the treatment of RC injuries, corroborating the present study, which demonstrated that exercise-associated KT significantly improved all movements tested in the study participants with right shoulder pain. Leong and $\mathrm{Fu}$ (42). approached 43 volleyball athletes through three taping protocols, with and without tension. The authors analyzed the subacromial space and observed that athletes with CR tendinopathy demonstrated less reduction in the subacromial space with rigid scapular taping during the early abduction of the arm (42). Tapings applied to the scapula showed no effect in the subacromial space during arm rest at $0^{\circ}$ of shoulder abduction (42). However, they showed less space reduction during active shoulder abduction from $0^{\circ}$ to $60^{\circ}$ in athletes with RC tendinopathy (42). This may indicate better control of the subacromial space during active arm abduction (42). The possible mechanism for the observed changes may be via skin stimulation. To support this assumption, is checked an earlier activation of the scapular muscles during the arm abduction and an increase in the lower trapezius activities during arm elevation (42). Therefore, therapeutic taping can improve neuromotor control of the scapular muscles for better control of the scapula and preserve the subacromial space during abduction of the arm (42).

Even if there is some clinical efficacy of applying adhesive tape, there is insufficient evidence of rehabilitation benefits to strongly recommend the application of KT in isolation (35).Other studies that investigate the effect of $\mathrm{KT}$ in the treatment of other joints, such as the knee, confirm that KT can support rehabilitation therapy. However, there are no significant effects in terms of recovery(35). 
More expressive results were obtained when associating KT with the exercise program, indicating important benefits from the optimization of exercises on the pain and function of these patients. Therefore, it seems to us that KT acts as an important adjunct in the recovery of rotator cuff injuries.

\section{STUDY LIMITATIONS}

Our study has some limitations that prevent the extrapolation of results. Initially, the sample - despite being calculated - is small. Moreover, a short follow-up period after the intervention prevents us from observing these results over time. We also chose to analyze the variables only including subjects with affected dominant and nondominant shoulder, which further reduces our sample, being a possible bias for extrapolating the results. The lack of a control or placebo group also hinders the extrapolation of findings. In addition, the lack of use of an isokinetic dynamometer to verify the strength of the study participants may be a limitation. This type of dynamometer has been cited by

\section{REFERENCES}

1. Oliveira FCL, Fontenay BP, Bouyer LJ, et al. Effects of Kinesiotaping Added to a Rehabilitation Programme for Patients with Rotator Cuff Tendinopathy: Protocol for a Single-Blind, Randomised Controlled Trial Addressing Symptoms, Functional Limitations and Underlying Deficits. BMJ. 2017;7(9):1-10.

2. Edwards A, Chepe J, Jones A, Sheps DM, Beaupré L. Can Clinical Assessment Differentiate Partial Thickness Rotator Cuff Tears from full Thickness Rotator Cuff Tears? A Secondary Analysis. Disabil Rehabil. 2019;8:1-8.

3. Saracoglu I, Emuk Y. Does taping in addition to physiotherapy improve the outcomes in subacromial impingement syndrome? A systematic review. Physiother Theory Pract. 2017;34(4):251-63.

4. Whittle S, Buchbinder R. In the Clinic. Rotator Cuff Disease. Ann Intern Med. 2015;162(1):1-15.

5. Steuri R, Sattelmayer M, Elsig S, et al. Effectiveness of conservative interventions including exercise, manual therapy and medical management in adults with shoulder Impingement: A Systematic review and meta-analysis of RCTs. Br J Sports Med. 2017;51(18):1340-47.

6. Clement ND, Nie YX, McBirnie JM. Management of degenerative rotator cuff tears: A review and treatment strategy. Sports Med Arthrosc Rehabil Ther Technol. 2012;48(4):1-5.

7. McLaren C, Colman Z, Rix A, et al. 2016. The Effectiveness of Scapular Taping on Pain and Function in People with Subacromial Impingement Syndrome: A systematic review. Int Musculoskelet Med. 2016; 38:(3):81-89.

8. Saraya S, Bakry RE. Ultrasound: Can it replace MRI in the evaluation of the rotator cuff tears? The Egyptian Journal of Radiology and Nuclear Medicine. 2016; 47(1): 193-201. some authors to be more reliable than the manual dynamometer, and thus could provide a more effective result for muscle strength.

Research Ethics Committee Opinion No. 2.152.768, Lutheran University of Brazil

\section{ACKNOWLEDGEMENTS}

We thank the Group of Studies and Research in Sports and Orthopedic Trauma Rehabilitation, Physiotherapy Course, Lutheran University of Brazil, Torres/RS/Brazil.

\section{CONCLUSIONS}

Exercises proved to be the basis of the treatment of rotator cuff injury. Associating KT with exercises significantly improved the function of patients with this type of injury. In isolation, KT was effective in reducing pain and function, but did not alter joint strength and mobility.

9. Mottram SL. Dynamic Stability of the Scapula. Man Ther. 1997; 2(3):123-31.

10. Kibler WB, Sciascia AD. What Went Wrong and What to do About It: Pitfalls In The Treatment Of Shoulder Impingement. In: Duwelius PJ, Azar FM, eds. Instructional course lectures, vol. 57, Rosemont: American Academy of Orthopaedic Surgeons, 2008;47:877-85.

11. Vieira FA, Olawa PJ, Belangero OS, et al. Lesão do Manguito Rotador: Tratamento e Reabilitação. Perspectivas e Tendências atuais. Rev Bras Ortop. 2015; 50(6): 647-651.

12. Camargo PR, Avila MA, Albuquerque-Sendín FA, et al. Eccentric training as a new approach for rotator cuff tendinopathy: Review and perspectives. Word J Orhtop. 2014; 5(5): 634-644.

13. Blume C, Price SW, Jackson ET, et al. Comparison of eccentric and concentric exercise interventions in adults with subacromial impingement syndrome. Int J Sports Phys Ther. 2015; 10(4): 441-55

14. Ortega-Castillo M, Medina-Porqueres I. Effectiveness of the eccentric exercise therapy in physically active adults with symptomatic shoulder impingement or lateral epicondylar tendinopathy: A systematic review. J Sci Med Sport. 2016; 19: 438-453.

15. Dejaco B, Habets B, Van Loon C, et al. Eccentric versus conventional exercise therapy in patients with rotator cuff tendinopathy: a randomized, single blinded clinical trial. Knee Surg Sports Traumatol Arthrosc. 2016: 28: 1-9.

16. Hallgren HB, Holmgren T, Öberg B, Adolfsson L, Johansson K, Adolfsson LE. A specific exercise strategy reduced the need for surgery in subacromial pain patients. Br J Sports Med. 2014;48: 1431-1436. 
17. Thelen MD, Dauber JA, et al. The Clinical Efficacy of Kinesio Tape for Shoulder Pain: A Randomized, Double-Blinded, Clinical Trial. J Orthop Sports Phys Ther. 2008; 38 (7): 389-95.

18. Kase K, Wallis J, Kase T. Clinical therapeutic applications of the Kinesio taping method. USA: Kinesio; 2013.

19. Oliveira FCL, Fontenay BP, Bouyer LJ, et al. Immediate Effects of Kinesiotaping on Acromiohumeral Distanceand Shoulder Proprioception in Individuals with Symptomatic Rotator Cuff Tendinopathy. Clin Biomech. 2019;1(61):16-21.

20. Seitz A. L, Podlecki L.A, Emily R. Melton E. R, et al. Neuromuscular Adaptions Following a Daily Strengthening Exercise in Individuals with Rotator Cuff Related Shoulder Pain: A Pilot Case-Control Study. IJSPT. 2019;1(14):74-87.

21. Kachingwe AF, Phillips B, Sletten E, et al. Comparison of Manual Therapy Techniques with Therapeutic Exercise In the Treatment of Shoulder Impingement: A Randomized Controlled Pilot Trial. J Man Manip Ther. 2008; 16(4): 238-47.

22. Wright AA, Wassinger CA, Frank M, et al. Diagnostic Accuracy of Scapular Physical Examination Tests For Shoulder Disorders: A Systematic Review. Br J Sports Med, 2013; 47(14):886-92.

23. Karthikean S, Griffin DR, Parson N, et. al. Microvascular Blood Flow in Normal and Pathologic Rotator Cuffs. J Shoulder Elbow Surg, 2015;24(12):1954-60.

24. Tantawy SA, Kamel DM. The effect of kinesio taping with exercise compared with exercise alone on pain, range of motion, and disability of the shoulder in postmastectomy females: a randomized control trial. J Phys Ther Sci. 2016;28(12):3300-05.

25. Michener LA, Walsworth MK, Burnet EN. Effectiveness of Rehabilitation for Patients with Subacromial Impingement Syndrome: A Systematic Review. J Hand Ther. 2004;17(2):152-64.

26. Ling SM, Conwit RA, Talbot L, et al. Electromyographic patterns suggest changes in motor unit physiology associated with early osteoarthritis of the knee. Osteoarthr. Cartil. 2007;15(10):1134-40.

27. Oku EC, Andrade AP, Stadiniky SP, et al. Translation and cultural adaptation of the Modified-University of California at Los Angeles Shoulder Rating Scale to portuguese language. Rev Bras Reumatol. 2006;46(4):246-52.

28. Martins J, Napoles BV, Hoffman CB, et al. The Brazilian version of shoulder Pain and Disability Index- translation, cultural adaptation and reliability. Braz J Phys Ther. 2010; 14(6): 527-536.

29. Oliva F, Piccirilli E, Bossa M, et al. I.S.Mu.L.T - Rotator Cuff Tears Guidelines. Muscles Ligaments Tendons J. 2016;5(4):227-63.

30. Kocyigit F, Acar M, Turkmen MB, et al. Kinesio taping or just taping in shoulder subacromial impingement syndrome? A randomized, double-blind, placebo-controlled trial. Physiother Theory Pract. 2016;32(7):501-8.

31. Ingwersen KG, Christensen R, Sorensen L, et al. Progressive High-Load Strength Training Compared with General
Low-Load Exercises in Patients with Rotator Cuff Tendinopathy: Study Protocol for a Randomised Controlled Trial. Trials. 2015;16(27):1-11.

32. Jaraczewska E, Long C. Kinesio Taping in Stroke: Improving Functional Use of The Upper Extremity in Hemiplegia. Top Sroke Rehabil. 2006;13(3):31-42.

33. Shakeri H, Keshavarz R, Arab AM, et al. Clinical effectiveness of kinesiological taping on pain and pain free shoulder range of motion in patients with shoulder impingement syndrome: a randomized, double blinded, placebo-controlled trial. Int J Sports Phys Ther. 2013;8(6) 800-810.

34. Thelen MD, Dauber JA, et al. The Clinical Efficacy of Kinesio Tape for Shoulder Pain: A Randomized, Double-Blinded, Clinical Trial. J Orthop Sports Phys Ther. 2008;38(7):389-95.

35. Miccinilli S, Bravi M, Morrone M, et al. A Triple Application of Kinesio Taping Supports Rehabilitation Program for Rotator Cuff Tendinopathy: a Randomized Controlled Trial. Ortop Traumatol Rehabil. 2018; 20(6):499-505.

36. Aguilar-Ferrándiz ME, Castro-Sánchez AM, Matarán-Peñarrocha GA, et al. A Randomized Controlled Trial of a Mixed Kinesio Taping-Compression Technique on Venous Symptoms, Pain, Peripheral Venous Flow, Clinical Severity and Overall Health Status in Postmenopausal Women with Chronic Venous Insufficiency. Clin Rehabil 2014;28(1):69-81.

37. Şahin O Ş, Biçer S, Şahin Z, et al. Effectiveness of Kinesiotaping and Subacromial Corticosteroid Injection in Shoulder Impingement Syndrome. Am J Phys Med Rehabil. 2016;95(8):553-60.

38. Kaya DO, Baltaci G, Toprak U. et al. The clinical and sonographic effects of kinesiotaping and exercise in comparison with manual therapy and exercise for patients with subacromial impingement syndrome: a preliminary trial. J. Manipulative Physiol Ther. 2014;37(6):422-32.

39. Şimşek HH, Balki S, Keklik SS, et al. Does Kinesio Taping in Addition to Exercise Therapy Improve the Outcomes in Subacromial Impingement Syndrome? A Randomized, Double-Blind, Controlled Clinical Trial. Acta Orthop Traumatol Turc. 2013;47(2):104-10.

40. Subaşı V, Çakır T, Arıca Z, et al. Comparison of efficacy of kinesiological taping and subacromial injection therapy in subacromial impingement syndrome. Clin Rheumatol. 2016;35(3):7416.

41. Hsu Y, Chen W, Lin H, et al. The effects of taping on scapular kinematics and muscle performance in baseball players with shoulder impingement syndrome. J Electromyogr Kinesiol. 2009;19(6):1092-9.

42. Leong HT, Fu SN. The Effects of Rigid Scapular Taping on the Subacromial Space in Athletes With and Without Rotator Cuff Tendinopathy: A Randomized Controlled Study. J Sport Rehabil. 2019 Mar 1;28(3):250-255.

43. Padulo J, Oliva F, Frizziero A, Maffulli N. Muscles, Ligaments and Tendons Journal - Basic principles and recommendations in clinical and field Science Research: 2018 update. MLTJ 2018; 8(3): $305-307$. 